\title{
Phase transfer measurements at the Jefferson Lab recirculated linacs
}

\author{
G.A. Krafft*, B.A. Bowling, M.T. Crofford, J.C. Hovater \\ Jefferson Lab, Newport News, VA 23606, USA \\ Available online 18 November 2005
}

\begin{abstract}
Bunch length or longitudinal phase space distribution measurements are often used to evaluate if the electron beam meets RF criteria for a recirculated linac. Unfortunately, in many instances when the beam fails to meet the needed criteria, such measurements provide little guidance as to which machine element is problematic. All of the Jefferson Lab recirculated linacs have employed longitudinal phase transfer measurement systems to provide both useful information on the initial understanding of the dynamics of the longitudinal phase space in these accelerators, and much more useful diagnosis of out-of-specification performance of machine RF elements. These systems can provide precision transfer function measurements in time scales convenient for machine operations.
\end{abstract}

(C) 2005 Elsevier B.V. All rights reserved.

PACS: 29.27.Bd; 29.27.Fh

Keywords: Phase transfer technique; Longitudinal beam dynamics; Bunch length; Recirculated accelerators; Energy recovered accelerators

\section{Introduction}

One primary determinant of the quality of the beam accelerated in the recirculated linacs at Jefferson Lab is the length of the accelerated bunches. Ideally, in the CEBAF accelerator one would like to obtain as short a bunch as possible both to reduce the energy spread in the emerging beam and to minimize transverse RF effects that can increase the emittance of the high-quality beam. Likewise, in the energy recovered free electron lasers (FELs) at Jefferson Lab smaller bunch length leads to higher peak current and higher FEL gain. Therefore, many studies on the bunch length have been completed at both the CEBAF accelerator and at the FELs, using both computer simulations and experiment. The main goals of these efforts were to quantify the smallest bunch length that could be obtained with the accelerators, and to develop and maintain good operational settings for them.

Early during the commissioning of the CEBAF accelerator, a generally useful concept was found and applied to develop and maintain the CEBAF settings, and it seems

\footnotetext{
*Corresponding author. TJNAF MS-7A, 12000 Jefferson Ave., Newport News, VA 23606, USA.

E-mail address: krafft@jlab.org (G.A. Krafft).
}

reasonable to summarize this idea at this workshop. Rather than measuring the bunch length or phase space distribution directly, but by measuring the linear and nonlinear longitudinal difference orbit in the regions of the accelerator important for bunching and debunching, one has the means of determining whether the bunching program has been properly executed. By this means one has the ability to rapidly explore the longitudinal bunching in the beam, and to diagnose and restore minimum bunch length configurations.

By examining the evolution and distortion of the longitudinal phase space as bunching occurs, it becomes clear that the bunch length is minimized not only when the phase space distribution is oriented without slope (i.e. there is a longitudinal focus), but also when some of the nonlinearity in the bunching force is systematically corrected by a suitable means. In the CEBAF accelerator this was accomplished by suitable phasing of the RF cavities producing the bunching [1,2] and in the free electron lasers the non-linearities were adjusted by incorporating higher order multipoles in the recirculation bends [3]. The procedure is somewhat analogous to the notion of making chromatic corrections in transverse optics, but where one tries to make time-of-arrival independent of starting energy or starting phase. Having a means to measure directly the 
non-linear longitudinal transfer characteristics of the bunching regions of the accelerator, as by phase transfer measurements, provides a means to rapidly determine and adjust the transfer characteristics to required values. In this paper we show the basic measurement system and provide examples and references to some experimental results from all of the Jefferson Lab recirculated linacs.

\section{Phase transfer measurement system}

The basic measurement system derives from a phase transfer function $\left(\mathrm{M}_{55}\right)$ measurement system that was built by Yao [4], but which has been systematically improved and extended over the years so that easy and reproducible measurements are rapidly possible [2,5,6]. The basic elements of the system are (1) a phase modulator, (2) longitudinal pickup cavities, and (3) a phase detector attached to the fundamental mode RF signals generated in the pickup cavities.

During the measurement, an intermediate frequency phase shifter is used to vary the phase of the RF field controlling the lasers which drive the photocathodes and produce the beam pulses. This has the effect of varying the emission time of the beam pulses with respect to all the other RF systems in the accelerator, which are nominally held constant throughout the duration of the measurement. The phase modulator is designed to have an offset phase that is a linear function of an applied control voltage. It has the capability to provide both sinusoidal phase modulation and triangular wave phase modulation with variable amplitude (this feature is only occasionally used) at frequencies around $70 \mathrm{~Hz}$, which is conveniently fast enough to allow a complete 1000 point measurement to be completed in 10s of seconds. In addition to having RF cavities for acceleration and bunching in the accelerators, longitudinal pickup cavities whose RF frequencies are tuned to the fundamental RF frequency or one of its low harmonics are installed at strategic locations throughout the accelerators for bunching diagnostics.

The diagnostic cavities are excited by the beam and the relative time-of-arrival is determined by RF phase detection with a mixer referenced to the master RF distribution system as shown in Fig. 1. This relative time-of-arrival monitoring gives the phase transfer function directly when both the modulation voltage and the phase detector voltage are simultaneously digitized with a transient recorder and the data is displayed on a control room display screen. Note that the phase detector output is first filtered and then amplified with a programmable gain amplifier (PGA), which allows one to increase the dynamic range in current for the measurement system. Within the phase detector chassis there is an electronic phase shifter on the master reference input to the mixer. The system is calibrated by varying the input phase of the reference across whole range of $360^{\circ}$ in the input phase, and recording the maximum and minimum output from the mixer with the beam current on. For phase detection the reference phase is left on the

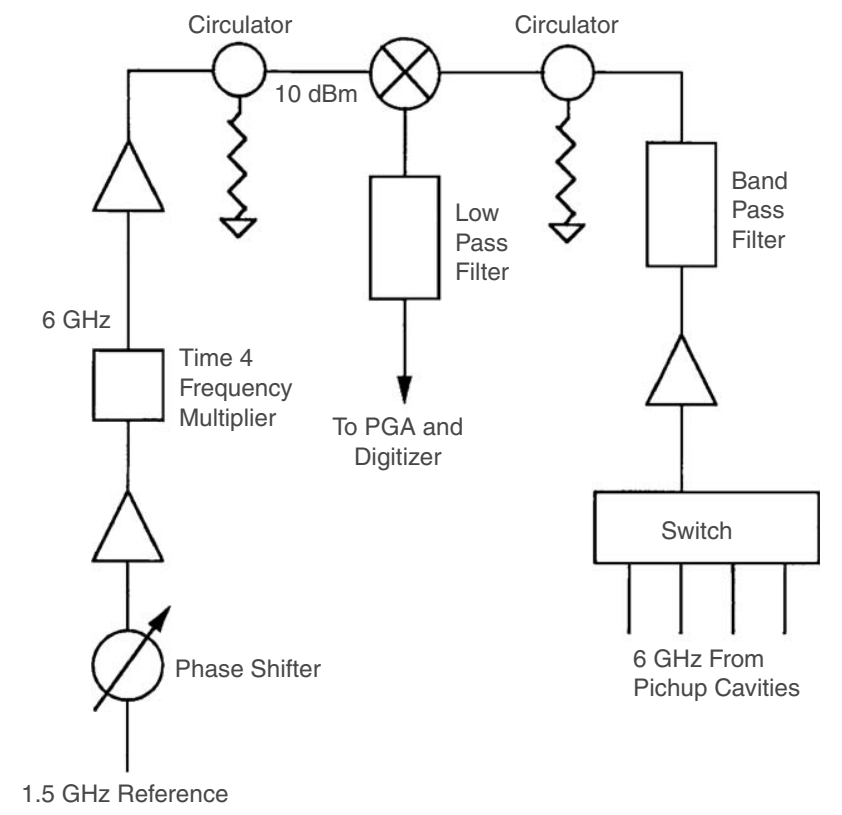

Fig. 1. Schematic of phase detector chassis for CEBAF phase transfer function measurement system.

zero crossing of the mixer output after the automatic calibration procedure is completed. Using presently available precision mixers, distortions in time-of-arrival at the $50 \mathrm{fs}$ level are easily discernible, with $25 \mu \mathrm{A}$ beam pulses of duration $100 \mu$ s. Given the high current present in the many possible applications discussed at this conference, even better accuracy is anticipated in such applications. Examples of phase transfer function measurements are shown in the next section.

\section{Examples of use}

Phase transfer function measurements have been used in all three of the recirculated linacs at Jefferson Lab. However, due to the broad applicability of the technique, the results of the measurements were used to investigate significantly different issues in the different machines.

The first measurement system was deployed in the earliest accelerator at Jefferson Lab, the CEBAF nuclear physics recirculated linac; one is interested in small bunch lengths in this accelerator to ensure superior beam quality in the extracted beam. In preparation for commissioning a series of numerical studies were done in order to determine the smallest bunch length possible from the injector design. The basic conclusions of these studies were that a compression from about 110 ps FWHM to about one-half a ps (a compression ratio of about 200) was possible in the design, and that the ultimate bunch length achieved would not be limited by the longitudinal phase space of the chopped beam coming from the electron source, but be limited by the non-linearities in the bunching process itself. Effort was spent designing settings that eliminated the first two orders of nonlinear phase space evolution 
in the injector. Information about the calculations, and the settings that produced short bunches is published elsewhere [2].

During CEBAF commissioning, a main task was to verify that short beam bunches could be obtained, and perhaps more importantly, to develop procedures to allow one to restore the bunch duration to small values when, because of accelerator system RF phase drifts (of course worst during the earliest period of accelerator commissioning), the bunch length grew to unacceptably large values. The design settings were established in the CEBAF injector and they yielded phase transfer measurements in beautiful agreement with the calculations $[1,7]$. Because the nonlinear transfer maps are sensitive indicators of RF phase errors in the accelerator system, it was natural to base the bunch length correction procedures on the appearance of the phase transfer maps as shown in Ref. [2]. As part of this effort, general methods to analyze nonlinear transfer maps in terms of their Tschebysev expansions were developed [8]. Because of the success of the phase transfer system in measuring small relative time changes, similar measurement systems were developed to measure and set overall recirculation path length [9] and the linear dependence of path length on energy $\left(\mathrm{M}_{56}\right)$ [10] of the recirculation arcs in the CEBAF accelerator.

The phase transfer technique was also extensively used during the commissioning and operation of the IRDEMO FEL at Jefferson Lab. In order to be able to energy recover the large energy spread of the electron beam generated by the FEL interaction, there is a bunch energy compression that is accomplished in the recirculation system of the energy recovered linac. Because the energy-compressed bunches must be decompressed in phase, the nonlinearities from the curvature of the RF deceleration during energy recovery become quite significant after energy compression. In order to correct the curvature, sextupoles were introduced in the bends of the recovery loop. Longitudinal RF cavities for the phase transfer measurements were placed on the linac axis and after each recirculation $180^{\circ}$ bend. As summarized in detail in Ref. [3], phase transfer measurements provided an important quantitative verification of the recirculation system design, and were essential in determining the correct recirculation path length setting for the single recirculation loop in that energy recovery linac. In contrast to the CEBAF accelerator where the measurements were done with 4th harmonic $6 \mathrm{GHz} \mathrm{RF}$ systems, the FEL systems were based on fundamental mode $1.5 \mathrm{GHz}$ RF systems. Because of the much higher average current of the FEL systems, no decrease in accuracy attended this change.

This same phase transfer measurement system is used in the higher power energy recovered FEL recently completed at Jefferson Lab. In Fig. 2 examples are given of two phase transfer functions [11]. The measurement is from the laser photocathode source up to the wiggler inside the straight section of the recirculation loop. The two figures correspond to two different settings of the sextupoles: the first
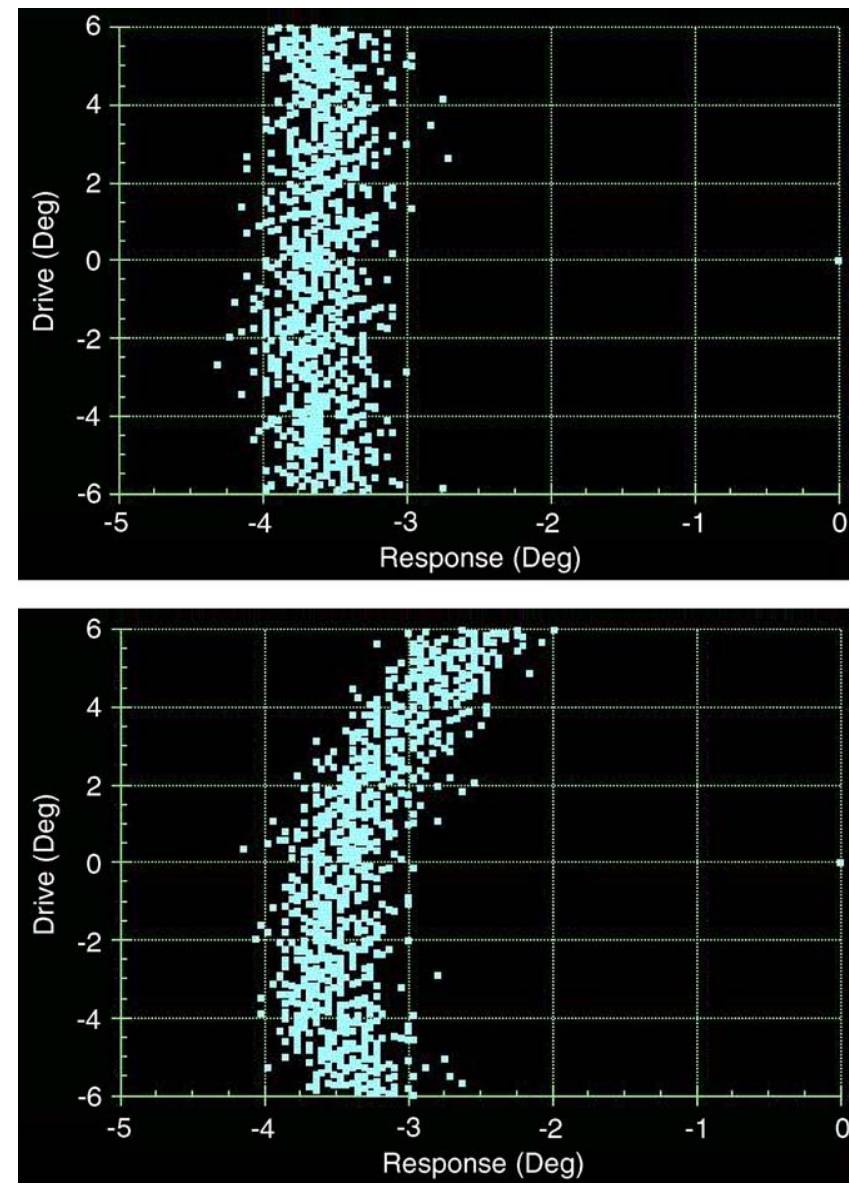

Fig. 2. Phase transfer function measurement at two sextupole settings in the Jefferson Lab energy recovered FEL [11].

has the nominal sextupole settings for the recirculator optics design, and the second one is at a setting of about $84 \%$ of nominal. To allow easier comparison to the longitudinal phase space underlying the distortion, the vertical axis gives the input phase and the horizontal axis gives the time-of-arrival as determined by the output from the phase detector. As is obvious from the measurement result, the nominal settings yield a time-of-arrival largely independent of emission phase. Whereas in the off nominal case, one observes the dominant $T_{566}$ distortion in the nonlinear transfer map which causes the middle of the phase space to arrive at times displaced away from the sides of the phase space.

\section{Conclusions}

In this paper the idea of the phase transfer function measurement has been introduced. Some examples of transfer functions measurements from the Jefferson Lab recirculated linacs have been presented and some of the commissioning uses of transfer function measurement systems have been discussed. The phase transfer function measurement technique was instrumental in allowing reproducible short bunches to be produced and maintained 
in these accelerators. It allowed beam based measurements of non-linear beam optical effects to be performed. The noise in the measurement technique is small enough that detailed information about the nonlinear longitudinal phase space evolution may be obtained. By applying the same techniques to the higher average current accelerators discussed at this conference, still better measurement resolution, at the level of $1-10 \mathrm{fs}$, should be possible.

\section{Acknowledgements}

This research was supported by the U.S. Department of Energy under Grant DE-AC05-84ER40150.

\section{References}

[1] G.A. Krafft, Proceedings of the 1994 Linac Conference, p. 9, 1994.
[2] G.A. Krafft, Proceedings of the Microbunches Workshop, AIP Conference Proceedings, vol. 367, 1995, p. 46

[3] P. Piot, D.R. Douglas, G.A. Krafft, Phys. Rev. ST-AB 6 (2003) 0030702.

[4] C.G. Yao, Proceedings of the 1990 Beam Instrumentation Workshop, AIP Conference Proceedings, vol. 229, 1990, p. 254.

[5] G.A. Krafft, Automatic phase transfer function measurements in the CEBAF front end, Presentation to the 1991 Beam Instrumentation Workshop, Unpublished.

[6] R. Abbott, B. Bowling, M. Crofford, J. Fugitt, C. Hovater, G. Krafft, Proceedings of the 1992 Linac Conference, p. 232, 1992.

[7] R. Abbott, et al., Proceedings of the 1994 Linac Conference, p. 777, 1994.

[8] G.A. Krafft, Proceedings of the 1997 Particle Accelerator Conference, p. 2268, 1997.

[9] G.A. Krafft, et al., Proceedings of the 1995 Particle Accelerator Conference, p. 2429, 1995.

[10] D. Hardy, et al., Proceedings of the 1997 Particle Accelerator Conference, p. 2265, 1997.

[11] D.R. Douglas, presentation at this conference. 